\title{
Coupling of low-frequency modes with the complex Ginzburg-Landau equation: Generalized Zakharov equations
}

\author{
R. Erichsen, L. G. Brunnet, and F. B. Rizzato \\ Instituto de Física, Universidade Federal do Rio Grande do Sul, Caixa Postal 15051, \\ 91501-970 Porto Alegre, Rio Grande do Sul, Brazil
}

(Received 21 June 1999)

\begin{abstract}
In this paper we introduce and examine a generalization of the complex Ginzburg-Landau equation (CGLE) where the self-interaction contained in the cubic term is replaced by a coupling involving the original field and a low-frequency one. New instabilities arise and a radically new asymptotic dynamical behavior emerges displaying defect turbulence over wide regions of the parameter space. [S1063-651X(99)00512-7]

PACS number(s): 05.45.Jn, 47.54.+r
\end{abstract}

The Zakharov equations (ZE for short) describe the slow modulational process arising in the nonlinear interaction of high- and low-frequency waves. The interaction of electronic and ion-acoustic waves in a plasma provides a good example of a process governed by the $\mathrm{ZE} \mathrm{[1].} \mathrm{In} \mathrm{its} \mathrm{original} \mathrm{form} \mathrm{the}$ $\mathrm{ZE}$ set is conservative, although not integrable [2]. Integrable dynamics can be obtained from the ZE if one makes some additional adiabatic assumptions on the dynamics. Then, the full set can be reduced to a nonlinear Schrödinger equation (NLSE) which can be fully integrated via inverse scattering [3]. From another perspective, the NLSE can also be seen as a particular form of the much more generic complex Ginzburg-Landau equation (CGLE) [4]. Unlike the NLSE, due to the complex character of the coefficients multiplying its various terms, the CGLE is nonconservative and models systems with sources and sinks. Precisely because of these features the CGLE is widely studied as a model equation in the field of dynamics of disordered regimes in spatially extended systems [4-7]. Extensive work by a number of authors shows that a variety of spatio-temporal patterns occurring in a wide range of physical settings can be understood with basis on the solutions of the CGLE [4-8]. Indeed, the universality of the CGLE allows its applicability to several nonlinear systems like wave interaction in fluids, laser propagation in nonlinear dieletrics like plasmas, as mentioned above, and many others [9].

The CGLE is an amplitude equation describing the dynamics close to a Hopf bifurcation of the relevant field. The bifurcation is assumedly saturated by a cubic term such that the final form of the equation is usually written in onedimension as

$$
\partial_{t} A=A+\left(1+i c_{1}\right) \partial_{x}^{2} A-\left(1+i c_{2}\right)|A|^{2} A
$$

$\left(\partial_{t, x} \equiv \partial / \partial(t, x)\right)$ with $c_{1}$ and $c_{2}$ real constants; symmetry is manifested by the invariance of the equation under $A$ $\rightarrow e^{i \varphi} A$ and $x \rightarrow-x$, where $\varphi$ is an arbitrary phase factor. We note that by taking the limit of large values of $c_{1}$ and $c_{2}$ one obtains the focusing or defocusing NLSE, all depending on the relative signal of the coefficients. The second spatial derivative present in Eq. (1) represents local coupling. Nonlocal coupling is to be considered when the shortest length scale of the fluctuations becomes smaller than the coupling range, what usually happens in situations of well developed turbulence $[10,11]$. However, since we are more interested in the circumstances leading to the turbulence we shall not go beyond a local approximation in the present work. As previously mentioned, Eq. (1) has been studied by a number of authors who mapped its basic behavior onto a $c_{1}-c_{2}$ parameter space. To remind the reader, we reproduce the parameter space in Fig. 1. The figure is relatively self-evident. However, we note that among all the curves present there, only one is analytical: the BFN (Benjamin-Feir-Newell) line. Bellow the BFN line there are stable attracting traveling waves (TW), and above the BFN line TW solutions become unstable. Those are the basic properties of the classical CGLE. We proceed now by pointing out that the nonlinear term of the CGLE is essentially a cubic one. This term satisfies all the symmetries demanded by the theory but provides only the lower order saturation for the Hopf bifurcation, certainly not the most generic one. In Ref. [12], for instance, the simple cubic interaction is replaced with more complicated cubic terms representing the self-interaction of a bidimensional vector field. Now in plasma physics or in laser-plasma interactions, for instance, the nonlinear term is not simply described by polynomial terms like the cubic term we have just mentioned. The nonlinear interaction arises as a result of the coupling of the slowly varying amplitude $A$ of a high-

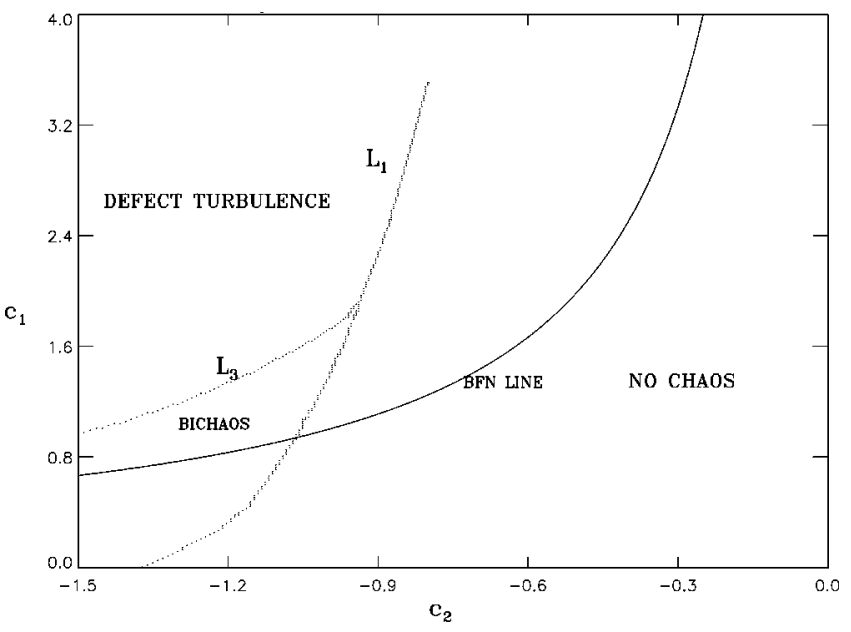

FIG. 1. The parametric $c_{1}$ vs $c_{2}$ plane and the various stable and unstable regions of the complex Ginzburg-Landau equation dynamics. See Ref. [6]. All quantities plotted are dimensionless. 
frequency field, with a low-frequency dynamical field, $\phi(x, t)$. Within the context of plasmas $\phi(x, t)$ represents low-frequency ion-acoustic fluctuations. In any case, when low-frequency dynamical fluctuations are present the appropriate set of governing equations describes the coupled dynamics of the slowly varying amplitude $A$ and the full field $\phi$ in the form

$$
\begin{gathered}
\partial_{t} A=A+\left(1+i c_{1}\right) \partial_{x}^{2} A+\left(1+i c_{2}\right) \phi A-c_{3} f\left(|A|^{2}\right) A, \\
\partial_{t}^{2} \phi-\partial_{x}^{2} \phi=\partial_{x}^{2}|A|^{2},
\end{gathered}
$$

which is a simple generalization of the conservative Zakharov equations, themselves obtained in the limit $\left|c_{1,2}\right|$ $\rightarrow \infty$ as mentioned earlier [2]. The ZE describe the dynamics within a fluid approximation, and nonconservative terms (energy sources and sinks) can be introduced and justified to model various processes connected to wave interaction with small scale granular structures like particles, for instance. This is the case of plasma physics, for example, where energy sources represent external beams of particles driving the relevant waves, and where energy sinks represent wave damping resulting from energy absorption by the plasma particles, the latter effect known as Landau damping [13-15]. In more specific terms, linear sources and sinks may be represented by the combination of the first and second terms on the right-hand-side of Eq. (2), which takes the form (1 $\left.-k^{2}\right) A$ for monochromatic waves if we note that $c_{1}$ is associated with nondissipative phase dynamics. This kind of combination yields amplification for small wave vectorswhich is the case when homogeneous beams drive plasma waves and dissipation for large enough wave vectorswhich can model linear Landau damping if we are a little economic and represent the symmetric damping in terms of quadratic terms in the wave vector. More complex, perhaps nonlocal, spatial dependences could be in principle treated with help of techniques discussed earlier [10,11]. Now the real component of the nonlinear term can model the nonlinear Landau damping, which occurs in addition to the linear damping when nonresonant wave-particle interaction is taken into account. All these features summed up, one may obtain a fairly global description of interactions in nonlinear continuous systems. Experiments on nonlinear wave in plasmas have been carried out during these last years and what has been detected is that under the combined action of amplification via beam driving and dissipation through linear and nonlinear Landau damping, turbulence does exist and is persistent over a wide range of the control parameters, from laboratory to space plasmas $[13,15,16]$. The kind of model studied here is suitable for these situations.

We shall refer to the set (2)-(3) as the generalized $\mathrm{Za}$ kharov equations (GZE), noting that we have augmented the equation for $A$ with a higher order nonlinear term that shall control the approach to saturation- $f(x) \sim x^{2}$ as $x \rightarrow 0$, which is higher order than the original cubic term, now replaced with the $\phi-A$ field coupling; $c_{3}$ is taken here as a real and positive coefficient and our results are highly insensitive on the particular form of $f(x)$. Let us stress the fact that the CGLE can be derived from the pair (2)-(3) if a quasi-static regime exists for which $\partial_{t}^{2} \phi \ll \partial_{x}^{2} \phi$. Then, from Eq. (3) one writes $\phi \sim-|A|^{2}$, and from Eq. (2) one thus obtains the CGLE.

In the nonchaotic region of Fig. 1, the pure CGLE generates only stable, attracting TW's. We found it convenient to emphasize this region in the present analysis, since it is precisely there that the contrast between the CGLE and GZE is sharper. In addition, since the dynamical behavior is similar over the entire CGLE regular region, we specialize our investigation entirely on the parametric point $c_{1}=c_{2}=0$.

We prepare the initial condition as follows: a stationary TW of the CGLE,

$$
A_{o}(x, t=0)=a_{o} e^{i k_{o} x}
$$

with $a_{o}=\sqrt{1-k_{o}^{2}}$, is added with a small random perturbing term of the form

$$
A_{1}(x, t=0)=a_{1}\left[\xi_{1}(x)+i \xi_{2}(x)\right] .
$$

As for the $\phi$ field we write

$$
\phi(x, t=0)=-a_{o}^{2}+c_{3} f\left(a_{o}^{2}\right),
$$

which guarantees a stationary solution if $a_{1} \rightarrow 0$. We take $a_{1} / a_{o}=0.01, k_{o}=7 k_{b}$ with $k_{b}$ as the basic wavevector used in the simulations- $k_{b}=2 \pi / L=0.01$ where $L$ is the system length, $\xi_{1,2}(x)$ as real uncorrelated random functions of the spatial coordinate satisfying $\left|\xi_{1,2}(x)\right|<1$, and $i^{2}=-1$. Were we working with the pure CGLE, perturbations would eventually vanish away and the final solutions would approach the TW asymptotic solution

$$
A(x, t)=a_{o} e^{i(k x-\Omega t)},
$$

with $\Omega=c_{2}-\left(c_{2}-c_{1}\right) k_{o}^{2}=0$ [4]. This is why the nonchaotic region is called so. But let us then examine gray level plots for $|A(x, t)|^{2}$ as generated by the GZE in Fig. 2; lighter and darker shades represent larger and smaller values of $|A(x, t)|^{2}$, respectively. Along with the parameters defined above, we use $f(x)=e^{x}-1-x$ (an exponential term provides fast saturation, but, as said earlier, other nonlinearities discussed in the literature generate similar results $[12,17]$ ), and $c_{3}=0.1$ in (a) and $c_{3}=1$ in (b). The solution initially looks like a traveling wave, but after a while, it diverges from that behavior. Several simulations were carried out with spectral and finite difference methods, both using discretizations with $N=1024$ units, but irrespectively of numerical procedures, all results agree and are conclusive: after an initial transient where the TW solution seems to be approached, the dynamics simply deviates from it into a highly turbulent motion preceded by the creation of relatively large scale structures which are decimated as time evolves. The presence of turbulence here is somewhat surprising, given the fact that in the non chaotic region of the CGLE one should perhaps expect a quasi-stationary regime allowing for the approximation $\phi \sim-|A|^{2}$, which in turn would lead to a plane wave solution, but now in the context of the GZE. An explanation is thus in order. The easiest way to see what is going on is to derive a dispersion relation that accounts for the linear stability of GZE plane waves. We perturb the plane wave (7) with a small field given by 
(a)

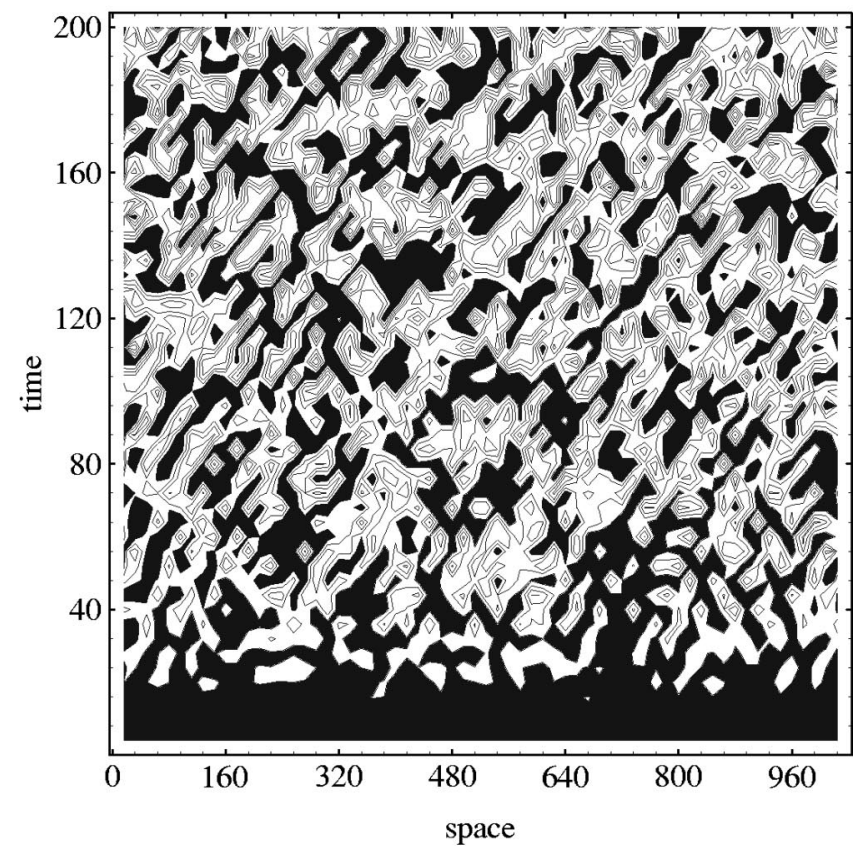

(b)

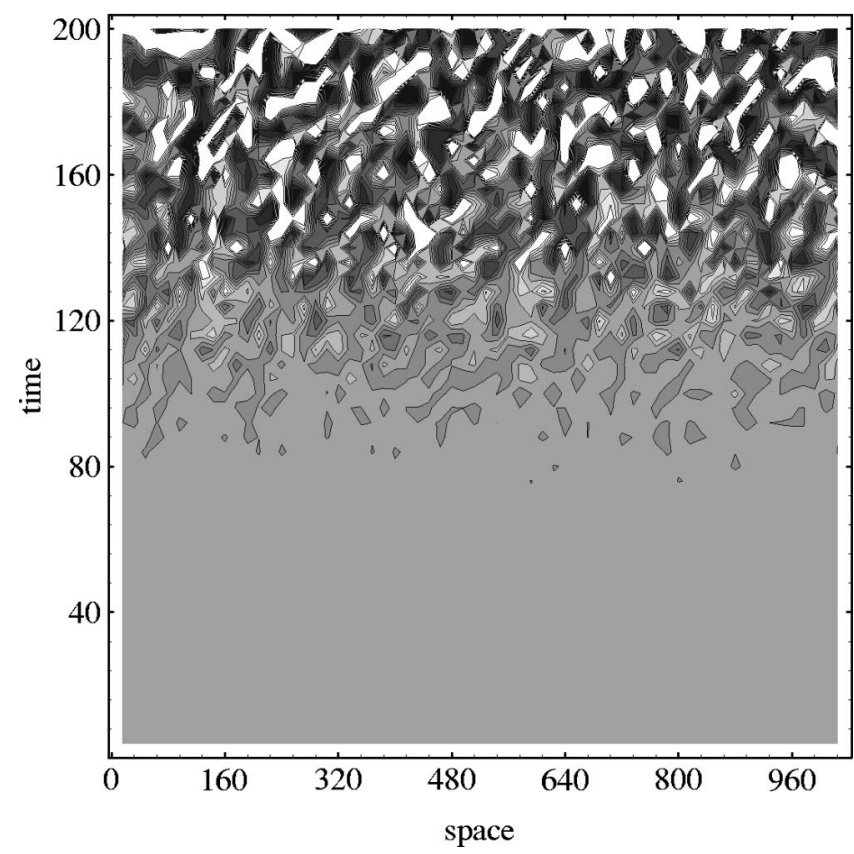

FIG. 2. Gray level plots for $|A(x, t)|^{2}$ as generated by random initial conditions evolved with the generalized Zakharov equations. $c_{3}=0.1$ in (a) and $c_{3}=1.0$ in (b). All quantities plotted are dimensionless.

$$
A_{1}(x, t)=a_{+}(t) e^{i\left(k_{o}+k\right) x}+a_{-}(t) e^{i\left(k_{o}-k\right) x},
$$

where $k$ is a continuous variable and $a_{ \pm}(t)$ are time dependent linear amplitudes of the form $a_{ \pm}(t) \sim e^{i \omega t}$, satisfying $\left|a_{ \pm}\right| \ll\left|a_{o}\right|$. The $\phi$ field is written as

$$
\phi(x, t)=-a_{o}^{2}+c_{3} f\left(a_{o}^{2}\right)+a_{\phi}(t) e^{i k x}+\text { c.c. }
$$

with $a_{\phi}(t)$ small, and where c.c. stands for complex conjugate. We point out that the basic interacting structure is con-

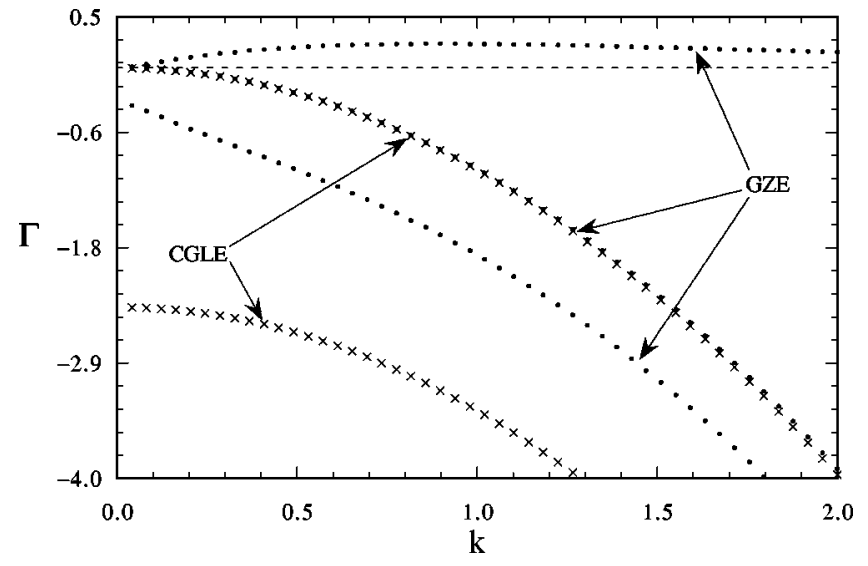

FIG. 3. Growth rates in the complex Ginzburg-Landau equation (CGLE) and generalized Zakharov equations (GZE) cases for $c_{3}$ $=0.1$. In contrast to the CGLE, one of the GZE roots is unstable. Horizontal dashed line indicates $\Gamma=0$. All quantities plotted are dimensionless.

stituted by a triplet of monochromatic waves in the $A$ field indexed by wave vectors $k_{o}$ - the pump wave-and $k_{o} \pm k-$ the daughter waves, plus a low-frequency mode with wavevector $k$. Then, from the corresponding linear analysis of Eqs. (2) and (3), a dispersion relation involving $\omega$ and $k$ is obtained:

$$
\begin{gathered}
{\left[\mathcal{D}_{\phi} \mathcal{D}_{+}(f)+k^{2}\left|a_{o}\right|^{2}\right]\left[\mathcal{D}_{\phi} \mathcal{D}_{-}(f)+k^{2}\left|a_{o}\right|^{2}\right]} \\
+\left[k^{2}+c_{3} \frac{d f}{d\left|a_{o}\right|^{2}} \mathcal{D}_{\phi}\right]^{2}\left|a_{o}\right|^{4}=0,
\end{gathered}
$$

where $\quad \mathcal{D}_{\phi} \equiv \omega^{2}-k^{2}, \quad$ and $\quad \mathcal{D}_{ \pm}(f) \equiv-i \omega \pm 2 k_{o} k+k^{2}$ $+d f / d\left|a_{o}\right|^{2}$. Should a quasi-static limit exist where $\omega^{2}$ $\ll k^{2}$, whenever $f=0$ the approximate relation could be written

$$
\left[\mathcal{D}_{+}(0)-\left|a_{o}\right|^{2}\right]\left[\mathcal{D}_{-}(0)-\left|a_{o}\right|^{2}\right]+\left|a_{o}\right|^{4}=0,
$$

which can be shown to be the CGLE dispersion relation indicating stability below the BFN line-note the CGLE dispersion relation is equivalent to the condition $\partial_{t}^{2} \phi \ll \partial_{x}^{2} \phi$ discussed earlier. However, numerical analysis of the GZE dispersion relation, Eq. (10), indicates that it has unstable solutions even below the BFN line, what in fact makes all the difference between the two cases. In Fig. 3 we represent not all the roots, but the various distinct growth rates calculated from Eqs. (10) and (11). One of the roots is almost identical in both cases and another, although not identical, is stable in both situations. But in contrast to the CGLE, one of the GZE roots is clearly unstable. When the off-equilibrium initial plane wave of the GZE tries to decay toward equilibrium, a small amount of radiation is produced in the $\phi$ field, a nonexistent feature of the CGLE scheme. The early dynamics is close to the one dictated by the CGLE, but eventually this small amount of radiation starts to act as a driver for Eq. (2) which feedbacks Eq. (3). The outcome of the process reverts in instability for the $\phi$ field and eventually for the whole dynamics. An investigation of relation (10) or an inspection of Fig. 3 shows that for the parameters of Fig. 2 (a), for instance, the fastest growth rate $\Gamma$ reads $\Gamma \sim 0.1$ 
which is compatible with the respective gray level plot displayed there. Another point worth of mentioning is that $\Gamma$ is always positive for the unstable root $(\Gamma \rightarrow 0$ only when $k$ $\rightarrow \infty$ ), which allows for the presence of a wide range of length scales in turbulent states.

Equally important is the final asymptotic state of the interaction. Further numerical analysis of the dispersion relation (10) reveals that the larger the coefficient of the saturating term, the smaller the growth rate. Comparison of Figs. 2(a) and 2(b) indeed shows that larger values of $c_{3}$ implies slower development of the instability; a more detailed analysis also indicates that slower instabilities are associated with lower levels of turbulence (in the present context, lower levels of turbulence means smaller maximum values for $|A|^{2}$ and $\phi$ ). On the other hand, the asymptotic steady states of both cases indicates a common feature: the modulus of the $A$ field hits the plane $|A|=0$ frequently and irregularly along temporal and spatial axes what enables to refer to the turbulence more specifically as a defect turbulence. The transient period before turbulence sets in can be more accurately described as a TW modulated by the presence of low-frequency modulations resulting from $\phi$ radiation, as had been already commented. Modulation can be more easily appreciated if one replaces the random functions used in the initial simulations with regular functions as follows:

$$
A_{1}(x, t=0)=a_{1}\left(e^{i\left(k_{o}+p k_{b}\right) x}+e^{i\left(k_{o}-p k_{b}\right) x}\right),
$$

where $p$ is an integer. Figure 4 considers the case $c_{3}=0.1$ and $p=3$. In the long run, the initial wave eventually evolves into a turbulent state as in the previous random cases. But in the transient TW period, Fig. 4(a), one can see more clearly the role of low-frequency fluctuations; the dynamics is modulated by the presence of fluctuations displaying precisely the spatial period of the low-frequency wave$p=3$ in the present situation. As mentioned above, it is the presence of the low-frequency radiation that provides the drive to turbulence. Details of the transition to turbulence are shown in Fig. 4(b); we again note that in the initial stages of the turbulent motion, a series of large localized structures are generated which are destroyed later on.

Defect turbulence does not occur under the BFN line of the CGLE case. The GZE therefore show some qualitatively new dynamics as compared to the CGLE. Now, a series of continuous systems supports low-frequency radiation like the one represented here by the $\phi$ field. In laser-plasma physics, as mentioned earlier, the ponderomotive potential of the high-frequency laser amplitude can easily drive lowfrequency waves through a coupling similar to what we use in this paper. What we show here is that the mere inclusion of this one additional degree-of-freedom alters the global behavior of the interaction; due to feedback processes, the presence of a dynamical low-frequency $\phi$ field leads to instabilities absent in the pure cubic case. Moreover, the saturation of the instabilities have also been discussed. After a period of time where the solution behaves like a TW modulated by the $\phi$ field, the dynamics evolves toward final turbulent states. The larger the coefficient of the saturating term, the longer the period where the dynamics resembles a TW one. The final asymptotic state, on the other hand, always displays defect turbulence with the modulus of the $A$ hitting the plane (a)

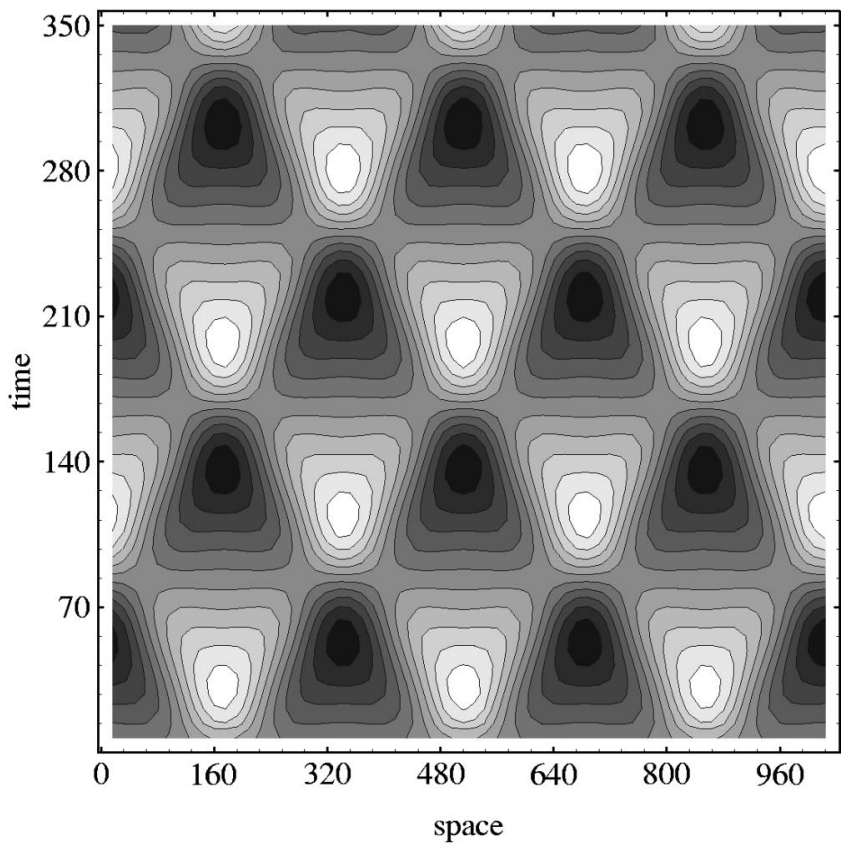

(b)

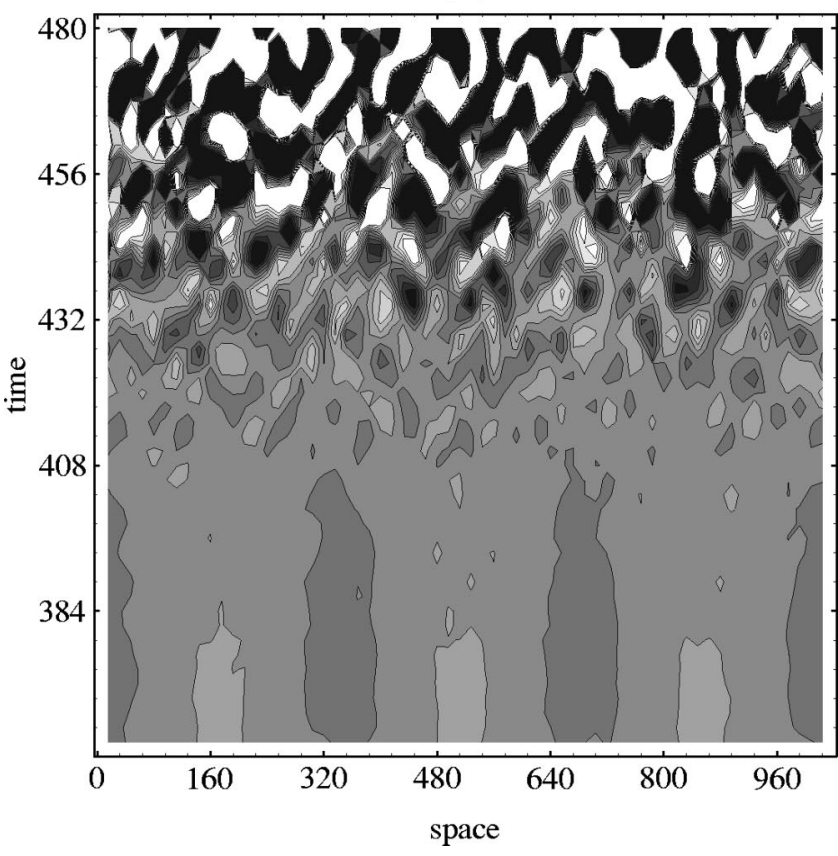

FIG. 4. Gray level plots for $|A(x, t)|^{2}$, with nonrandom initial conditions evolved with the GZE. $c_{3}=0.1, p=3$ and remaining parameters as in Fig. 2. A short run in (a) and a window showing the transition to turbulence in (b). All quantities plotted are dimensionless.

$|A|=0$ repeatedly. We have not analyzed in full details the dynamics in those regions of the parameter space where the dynamics arising from the CGLE is already chaotic. However, initial investigations show that for moderate values of $c_{1}$ and $c_{2}$, there is no appreciable differences between those cases and the particular situations analyzed here: in all cases a transient TW evolves into spatio-temporal chaotic patterns revealing defect turbulence. As low-frequency fluctuations are actually a frequent feature of spatially extended systems, the subject deserves some attention. 


\section{ACKNOWLEDGMENTS}

This work was partially supported by Financiadora de Estudos e Projetos (FINEP) and Conselho Nacional de De- senvolvimento Científico e Tecnológico (CNPq), Brazil. Numerical computing was partially performed on the CRAY Y-MP2E at the Universidade Federal do Rio Grande do Sul Supercomputing Center.
[1] S. G. Thornhill and D. ter Haar, Phys. Rep., Phys. Lett. 43C, 43 (1978).

[2] R. Erichsen, G. I. de Oliveira, and F. B. Rizzato, Phys. Rev. E 58, 7812 (1998).

[3] M. Ablowitz and H. Segur, Solitons and the Inverse Scattering Transform (Siam, Philadelphia, 1981).

[4] R. Montagne, Ph.D. thesis, Universitat de les Illes Balears, Spain, 1997.

[5] B. I. Shraiman, A. Pumir, W. van Saarloos, P. C. Hohenberg, H. Chaté, and M. Holen, Physica D 57, 241 (1992).

[6] H. Chaté, Nonlinearity 7, 185 (1994).

[7] M. Cross and P. Hohenberg, Rev. Mod. Phys. 65, 851 (1993).

[8] L. G. Brunnet, H. Chaté, and P. Manneville, Physica D 78, 141 (1994).

[9] P. Coullet, D. Daboussy, and J. R. Tredicce, Phys. Rev. E 58, 5347 (1998).
[10] Y. Kuramoto, Prog. Theor. Phys. 94, 321 (1995).

[11] Y. Kuramoto and H. Nakao, Phys. Rev. Lett. 76, 4352 (1996).

[12] E. Hernandez-Garcia, M. San Miguel, M. Hoyuelos, and P. Colet, in Europhysics Conference Abstracts, edited by P. L. Garrido and J. Marro (European Physical Society, Paris, 1998), Vol. 22F, p. 59.

[13] M. V. Goldman, Rev. Mod. Phys. 56, 709 (1984).

[14] G. D. Doolen, D. F. DuBois, and H. A. Rose, Phys. Rev. Lett. 54, 804 (1985).

[15] S. E. Gibson, D. L. Newman, and M. V. Goldman, Phys. Rev. E 52, 558 (1995).

[16] N. A. Krall and A. W. Trivelpice, Principles of Plasma Physics (McGraw-Hill, New York, 1973).

[17] P. Marcq, H. Chaté, and R. Conte, CNLS Nonlinear Science, e-print, patt-sol@xyz.lanl.gov 93100004. 